\title{
Competencias de Comunicación y Liderazgo en el grado de Comunicación Audiovisual
}

\author{
María Fernanda Gambarini Duarte, Ana Del Valle Morilla ${ }^{b}$, Jose María Alejos Bonilla ${ }^{c}$ \\ Ricardo León Fernandez \\ ${ }^{a}$ Universidad Francisco de Vitoria, Madrid, España, m.gambarini@ufv.es, ${ }^{\text {b }}$ Universidad Francisco de \\ Vitoria, Madrid, España, a.delvalle@ufv.es, ${ }^{C}$ Universidad Francisco de Vitoria, Madrid, España, \\ jm.alejos@ufv.es, ${ }^{\mathrm{d}}$ Universidad Francisco de Vitoria, Madrid, España, r.leon@ufv.es.
}

\begin{abstract}
Resumen
La pretensión de esta comunicación es mostrar la experiencia educativa transversal en la adquisición de competencias con alumnos de primer curso del grado de Comunicación Audiovisual. Se trata de una experiencia enmarcada en una praxis amplia de proyectos transversales en la Universidad Francisco de Vitoria, que responde a la voluntad de diálogo entre las disciplinas y a una misión compartida de formación integral del alumno.
\end{abstract}

Palabras clave: Competencias, liderazgo, acompañamiento, comunicación, formación integral.

\section{Introducción}

El ser humano es social por naturaleza y por necesidad morfologíca y constitutiva o natural; necesita del otro para desarrollarse. La forma fundamental que tiene de relacionarse con los demás, para responder a esa necesidad natural de relación, es la comunicación. Por lo tanto, se hace en tanto que se comunica y se hace de una u otra manera en función de la riqueza de sus comunicaciones, de la fortaleza de los vínculos que crea y del sentido que aportan a su vida estas interacciones.

La vía humana para la comunicación es el lenguaje, y el medio de transmisión fundamental en el siglo XXI de ese lenguaje, son los medios audiovisuales (actualmente adquieren gran relevancia los medios de comunicación social y las redes sociales en el mapa de la comunicación humana). Pero para que dicho lenguaje sea una vía de comunicación eficaz, constitutivo de contenidos audivisuales con sentido técnico, ético y estético, debe cumplir con unos requisitos. Requisitos, por otro lado demandados, por la industria audiovisual. Además, si no se pierde de vista el impacto 
real que los medios de comunicación tienen sobre la vida económica, política y personal de la comunidad, y se adopta una perspectiva integradora, esos contenidos deberían dar respuesta a la responsabilidad inherente a su impacto e incidencia en la sociedad. Una responsabilidad artística (saber y saber hacer en relación a unos estándares de calidad); una responsabilidad económica (saber hacer generando beneficios económicos) y una responsabilidad social (saber ser adecuada fuente de educación informal de la sociedad)

Esa responsabilidad social inherente a la profesion del comunicador audivisual queda perfectamente reflejada en las palabras de Puttnam «No creo que una película sola ni un solo artículo periodístico cambien nada. Pero con el paso de los años, el continuo goteo de buenas películas y de abundantes artículos, la calidad de los periódicos y el temple e integridad de los directores de periódicos, sí tienen gran importancia. El efecto de ese goteo es una dieta diaria de visiones e ideas a las que adherirse y que promueven lo mejor que hay en la sociedad; todo eso tiene un efecto. No simplemente una película, o un artículo, sino el hecho de que todos nosotros nos empeñemos de verdad en ser mejores y en funcionar mejor ${ }^{1}$

Ello demanda, que los profesionales que se dedican a la elaboración y gestión de contenidos audiovisuales, cuenten con una serie de competencias técnicas y personales que les habiliten para generar contenidos que promuevan lo mejor que hay en la Sociedad (el bien común).

A tal fin se ha planteado en el primer curso del grado de Comunicación audiovisual una experiencia educativa transversal entre las asignatura de Habilidades para la comunicación y el liderazgo, Tecnología Multimedia e Introducción a los Estudios Universitarios. Se plantea un acompañamiento coordinado desde las tres asignaturas en la realización de un proyecto con metodología colaborativa para el diseño y elaboración de una propuesta para canal de televisión con calidad ética, técnica y estética.

\footnotetext{
${ }^{1}$ Puttnam, D., “Filmmakers are missing their social purpouse”, Los Angeles Times, Mayo, 1988, p. 7.
} 


\section{Desarrollo}

La implementación de esta experiencia educativa en el primer curso, responde a la pretensión de que el proyecto sea una Estación Cero; un escenario de simulación de trabajo en equipo de producción audiovisual, que favorece el entrenamiento y desarrollo de competencias de trabajo en equipo y liderazgo, que puedan ser banco de pruebas para una experiencia posterior de proyectos transversales vinculados a empresas, durante los cursos superiores.

En dicha linea, desde los ámbitos de las distintas disciplinas involucradas; pero siempre de manera coordinada se ha acompañado a los alumnos de la siguiente forma:

La asignatura de Habilidades para la Comunicación y el liderazgo se ha encargado específicamente de favorecer el desarrollo de habilidades y actitudes intrapersonales e interpersonales imprescindibles para trabajar eficazmente en un equipo de producción en contenidos audiovisuales. Dada la dimensión social del ser humano a la que se hacía referencia en la introducción de este trabajo, cada una de las acciones que la persona realiza, repercute de manera más o menos directa en la vida de otras personas singulares y en el conjunto de la sociedad. Por ello se ha de contemplar la acción del comunicador audiovisual en la creación de contenidos audiovisuales desde la perspectiva del impacto que dicha acción tiene sobre la sociedad.

En el contexto actual, los medios audiovisuales ya no se surrogan a la defición que hace 30 años se hacía de ellos en términos de neutralidad, independencia o simples canales de distribución de la información. En la actualidad, como afirma Benavides (2008), los medios de comunicación junto con las instituciones y los colectivos sociales son instrumentos configuradores de los escenarios donde se mueve la ciudadanía. Ante esa realidad el comunicador audiovisual debe contar con una serie de competencias personales que encaminen su acción creadora y de gestión en la línea de la definición de producción audiovisual de calidad acuñada por LAICOM": "Una producción audiovisual de calidad es aquella que contribuye a mejorar la calidad de vida y, por tanto: al fomento del bienestar emocional, de las relaciones interpersonales, de los derechos, de la inclusión social y del desarrollo de las facultades intelectuales de sus consumidores"

Para ello, desde la asignatura de Habilidades para la Comunicación y el liderazgo, con un acompañamiento individual y grupal con los alumnos de primero de grado, se ha promovido el desarrollo de habilidades asociadas al trabajo en equipo y a la competencia de liderazgo.

\footnotetext{
${ }^{2}$ LAICOM (Laboratorio de Análisis Instrumental de la Comunicación. Universidad Autónoma de Barcelona)
} 
La asignatura de Tecnología Multimedia ha procurado combinar la formación técnica (los medios necesarios para la producción del mensaje audiovisual), con la estética (la adecuación de esos medios) mediante la formación de un criterio riguroso de pensamiento en los alumnos. Se ha intentado desarrollar competencias de toma de decisiones y trabajo en equipo desde el área de formación de las destrezas en el manejo de los softwares y la coordinación de los equipos de trabajo para la producción de audiovisuales.

Desde un razonamiento abierto de la Tecnología multimedia, se ha intentado reconducir la tecnocracia hacia un discruso de medios y fines. Así el fin es siempre la voluntad creativa y estética del comunicador y los diversos medios tecnológicos sirven a esta.

En el Proyecto Estación Cero, ha dotado de los conocimientos técnicos y las habilidades prácticas para realizar un inventario de medios técnicos y unos recursos humanos adecuados. También ha fomentado la reflexión sobre el uso de estos medios, desde un capítulo introductorio de identidad visual .

«El análisis de las tecnologías no puede hacerse al margen de las culturas que las originan (...) Las técnicas y tecnologías (todas ellas), permiten el trato del hombre con el mundo y, en ese sentido, son también medio en el que se nos hace presente alguna dimensión del ser ${ }^{3}$

Este punto que destaca Abellán-García en su propuesta de una Teoría dialógica de la Comuncación, es el nexo que vincula el Proyecto, Si el foco está en el alumno y su aprendizaje, los procesos de trabajo en equipo y el desarrollo de las competencias de comunicación y trabajo en equipo, se encuentra con la adecuación en la elección de medios tecnológicos para la producción de un canal audiovisual que, como todo buen producto audiovisual, está llamado a hacer inteligible o habitable o bello el mundo.

La asignatura de Introducción a los Estudios Universitarios se ha dedicado a profundizar en los criterios ético-estéticos de un producto audiovisual de calidad. Esta asignatura, dotada de un carácter propedéutico, busca salir al encuentro de la realidad del alumno universitario de primero de grado y su objetivo fundamental es hacerle consciente del verdadero sentido del ser universitario como buscador de la verdad. Para que está búsqueda sea plenamente universitaria, Introducción a los Estudios

\footnotetext{
3 ABELlÁN-GARCÍA, A., Crítica, fundamentos y corpus disciplinar para una Teoría dialógicca de la comunicación, Fundación Universitaria Española, Madrid: 2012, 331-332
} 
Universitarios, se centra en el desarrollo de las herramientas propias del trabajo intelectual.

A la formación integral que buscamos en cada alumno, sirve desde esta dimension del conocimiento en el esquema competencial.

Al Proyecto Estación Cero, le dota del desarrollo de competencia de investigación para la relfexión individual y para la elección del tipo de Proyecto. También busca formar la conciencia ética que ayude a responder a nuestros alumnos a la pregunta : “¿Qué tipo de productos audiovisuales necesita la comunidad la que me dirijo, siendo consciente de que uno de los fines de la television es el servicio público, expresado habitualmente en el Segundo de sus grandes objetivos : Informar, Formar y Entretener.

Además, Introducción a los Estudios Universitarios, propone una lectura de la realidad en ámbitos, a través, principalmente de la Obra de Alfonso López- Quintás. Así, la dimension estética de todo audiovisual adquiere un caracter ambital que invita al encuentro comunicativo que se indica en la introducción de esta comunicación.

«Este descubrimiento preciso de la condición ambital de ciertas realidades encierra la mayor importancia por una razón decisiva: los ámbitos pueden encontrarse entre sí y los objetos no. Y ya sabemos que el ser humano vive como tal, se desarrolla y perfecciona creando encuentros de uno y otro orden» ${ }^{4}$.

Desde este planteamiento transversal, el proyecto se estructura en las siguientes fases:

1. Fase de investigación y propuesta individual: de manera individual, el alumno tiene que documentarse y explicar la realidad de la producción televisiva del siglo XXI. A partir de la realidad señalada, debía identificar las principales preocupaciones que muestra la audiencia con respecto a esos contenidos. Establecido el contexto se proponen unos contenidos televisivos de calidad.

2. Fase de elaboración del Proyecto: a partir de esta fase, el trabajo es fundamentalmente en equipo. A través una ficha que se proporciona al alumno se sintetiza la información del Proyecto.

\footnotetext{
4 LÓPEZ-QUINTÁS, A.,Cómo formarse en Ética a través de la Literatura, Rialp, Navarra, 2007, 30.
} 
Tabla 1. Ficha cuadro síntesis del Proyecto

\begin{tabular}{|c|l|l|}
\hline \multicolumn{2}{|l|}{ PROPUESTAS CONTENIDOS TELEVISIVOS: (Tipo) } & $\begin{array}{l}\text { ALUMN@: (Nombre y apellidos del } \\
\text { alumn@ que hace la propuesta) }\end{array}$ \\
\hline $\begin{array}{c}\text { JUSTIFICACIÓN } \\
\text { (Se explica el porqué y el para qué de la propuesta de cambio o mejora en cuanto a } \\
\text { contenidos televisivos, en base a la situación real en la España del siglo XXI) }\end{array}$ \\
$\begin{array}{c}\text { DESCRIPCIÓN } \\
\text { DE LOS } \\
\text { PROPUESTOS }\end{array}$ & (Características, valores estándar de calidad a considerar, público objetivo...) \\
\hline $\begin{array}{c}\text { OBJETIVOS } \\
\text { CONSEGUIR }\end{array}$ & $\begin{array}{l}\text { Claros, concretos y coherentes con el proyecto. No deben ser más que un objetivo } \\
\text { general y tres objetivos específicos. }\end{array}$ \\
\hline
\end{tabular}

3. Fase de desarrollo del Proyecto: esta fase consiste en llevar a la acción la propuesta del equipo. Se elabora el borrador del proyecto en base a unos criterios de calidad, elección de localizaciones, salas, equipo humano y técnico. Se procede también al rodaje, producción y postproducción. Se realiza la grabación, edición o realización, sonorización, postproducción y finalización del producto audiovisual en formato de entrega (DVD o similar correctamente finalizado, etiquetado...), con la inclusión de títulos de crédito y revisión.

4. Fase de presentación y defensa del Proyecto: cada equipo entrega el producto audiovisual en el formato elegido. y la memoria final en la que queda recogido todo el proceso de elaboración del proyecto y del trabajo de equipo. Posteriormente, se presenta y defiende de la propuesta de canal de televisión.

\section{Objetivos}

3.1. Objetivos generales:

Aplicar los valores y competencias propias de la profesión del comunicador audiovisual.

Demostrar el manejo de las técnicas y herramientas de comunicación eficaz en la relación de equipos de trabajo.

3.2. Objetivos específicos:

$\checkmark \quad$ Extraer información relevante sobre la situación de la oferta televisiva del siglo XXI. 
$\checkmark \quad$ Analizar los criterios de calidad en la producción televisiva.

$\checkmark \quad$ Identificar las necesidades en cuanto a producción de contenidos.

$\checkmark \quad$ Diseñar y planificar una propuesta de un canal de televisión creativo que responda a criterios de calidad ética, técnica y estética.

\section{Metodología}

Se ha combinado la clase magistral, la flippedclasroom, la herramienta educativa de la gammificación con apoyo de un entorno virtual b-learning. A través de la plataforma Wikispaces, se asigna una wiki a cada equipo y se van regulando las distintas fases del Proyecto.

El producto que se recoge a partir de dichas fases son el resultado de investigación individual y grupal de los alumnos a partir de videos, artículos, miniquest, así como encuestas elaboradas y realizadas por los propios alumos.

\section{Resultados}

Se presentan los resultados obtenidos (gráfico 1) de un cuestionario realizado a los alumnos a final de curso en que debían valorar en qué medida la realización del Proyecto transversal ha contribuido al desarrollo de habilidades asociadas a la comunicación (tabla 1) dentro de un equipo de trabajo.

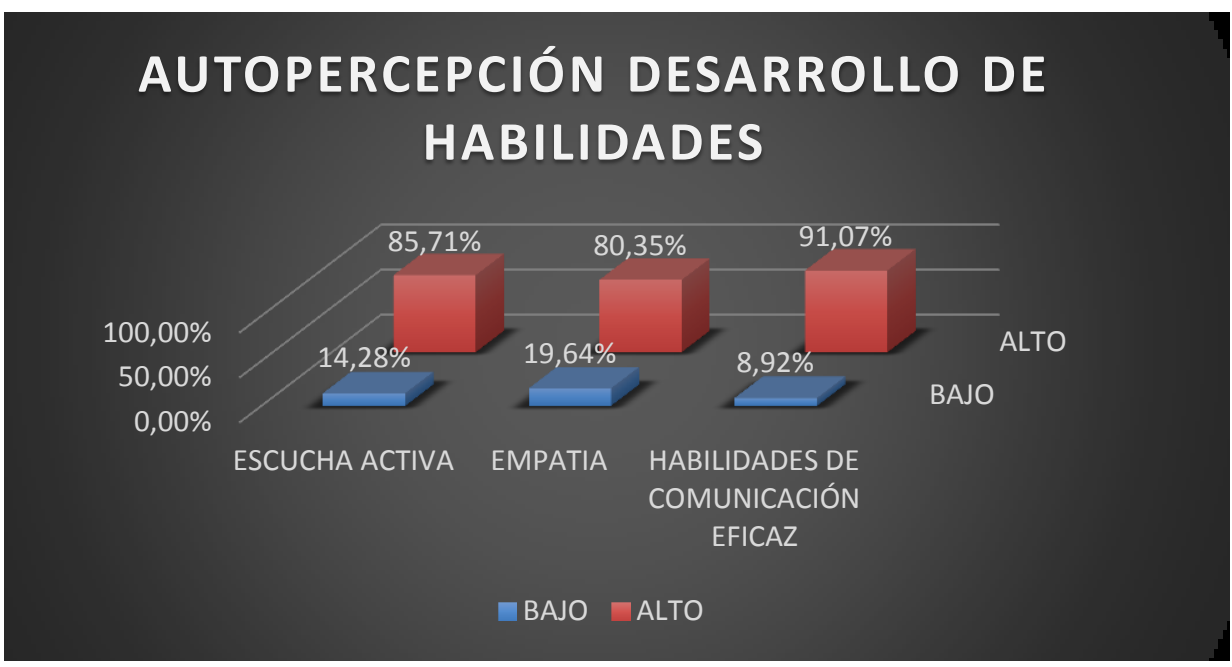

Gráfica 1. Cuestionario autoevaluación desarrollo competencial. Fuente: elaboración propia. 
Las habilidades en las que se ha pedido a los alumnos que evaluaran su nivel de desarrollo estuvieron medidas en función de una serie de capacidades a partir de conductas específicas (Tabla 1)

Tabla 1. Cuestionario autoevaluación desarrollo competencial . Fuente: elaboración propia

\begin{tabular}{|c|l|}
\hline \multirow{2}{*}{ ESCUCHA } & $\begin{array}{l}\text { Item 1: Capacidad de escuchar sin interrumpir . } \\
\text { Item 2: Capacidad para attender al lenguaje no verbal del otro. } \\
\end{array}$ \\
& $\begin{array}{l}\text { Item 3: Capacidad para resumir el mensaje del emisor . } \\
\text { Item 4: Capacidad para solicitar ampliacion de información del emisor. }\end{array}$ \\
\hline \multirow{2}{*}{ EMPATÍA } & Item 1: Capacidad de comprender la situación del otro. \\
& Item 2: Capacidad para tener en cuenta sentimientos del otro. \\
& Item 3: Capacidad de comunicación empática. \\
& Item 4: Capacidad de reflexión sobre impacto propia acción en el otro. \\
\hline COMUNICACIÓN & Item 1: Actitud apertura y flexibilidad ante la opinion del otro. \\
& Item 2: Voluntad de comunicación eficaz . \\
& Item 3: Capacidad de adaptar el estilo de comunicación. \\
& Item 4: Capacidad de comunicación clara, sincera y efectiva. \\
\hline
\end{tabular}

\section{Conclusiones}

Por un lado, desde la valoración por parte del equipo docente que ha coliderado esta experiencia educativa se concluye que dicha experiencia ha servido a los alumnos para enfrentarse a una situación pseudoreal de creación y gestión de un producto audiovisual, la cual ha favorecido el inicial desarrollo de competencias técnicas propias de la ideación y creación de un producto audiovisual y trasnversales de trabajo en equipo imprescindibles en su futuro profesional.

Por otro lado, los resultados del cuestionario realizado a los alumnos a final de curso recogidos en la gráfica 1, ponen de manifiesto una percepción, por parte de éstos, de mejora que coincide con la observación realizada por el equipo docente en cuanto al ambiente de trabajo dentro de los equipos, así como también en la calidad de los productos presentados.

Por tanto, los autores de esta comunicación consideran que la experiencia educativa transversal, Proyecto Estación Cero, ha procurado combinar la formación técnica, con la estética mediante la formación de un criterio riguroso de pensamiento en los alumnos. Se les ha dotado de los conocimientos técnicos y las habilidades prácticas para realizar un inventario de medios técnicos y unos recursos humanos adecuados, al tiempo que se ha fomentado la reflexión sobre el uso de estos medios y de la responsabilidad social inherente a la creación de los productos audiovisuales. Con todo ello, usando una metodología de trabajo cooperative, se ha favorecido la puesta en juego de habilidades de comunicación eficaz y de trabajo de equipo entre los alumnos. 


\section{Referencias}

Abellán-garcía, A. (2012) Crítica, Fundamentos y corpues disciplinar para una Teoría dialógica de la Comunicación, Fundación Universitaria Española, Madrid.

Álvarez De Mon, S. (2004) Carácter del liderazgo: Lecciones desde la adversidad. Revista Empresa y Humanismo Vol. V, n 1/02, , pp. 23-46 Fecha de consulta: 18 de Junio de 2018 (https://goo.gl/P3xn3Q)

Bañares, L El carácter sistémico del liderazgo. Anuario Filosófico 1996, n 26, p 386 Servicio Publicaciones Universidad de Navarra. Pamplona. Fecha de consulta: 20 de Junio de 2018 (https://goo.gl/oChJZC)

Agejas, J. (2013) La ruta del encuentro, Universidad Francisco de Vitoria, Madrid.

Benavides Delgado2, J. (2008). Los medios de comunicación en la actual coyuntura: la necesidad de rectificar. Pensar La Publicidad. Revista Internacional De Investigaciones Publicitarias, 2(1), 79 - 92. doi:10.5209/PEPU.16391 Fecha de consulta: 18 Junio 2018. (https://goo.gl/aVZCdM)

Cardona, P y García-Lombardia, P. (2005). Cómo desarrollar las competencias de liderazgo. Libros IESE. EUNSA. Pamplona.

Cardona, P., Rey, C., El liderazgo centrado en la misión: Cómo lograr el liderazgo en toda la organización, IESE, OP-08-4, 01/2008, p. 2. Fecha de consulta: 18 de Junio de 2018 (https://goo.gl/e2mRnG)

Delgado Fernández, M., \& Solano González, A. (2009). Estrategias didácticas creativas en entornos virtuales para el aprendizaje. Revista Electrónica "Actualidades Investigativas en Educación", 9 (2), 1-21. Fecha de consulta: 29 de Junio de 2018 (https://goo.gl/v41F7J)

Finkel, D. (2008) Dar clase con la boca cerrada, PUV, Valencia.

Lopez Quintás, A. (1998) Estética de la Creatividad, Rialp, Madrid,

Pardo, A., (2001): "El cine como medio de comunicación y la responsabilidad social del cineasta”, en Codina, M. (ed.): De la ética desprotegida. EUNSA, Pamplona, pp.117-141.

Tarkovski, A. (2016) Atrapad la vida. Lecciones de vida para escultores en el tiempo. Errata Naturae, Madrid. 